\title{
Comparative transcriptome investigation of Nosema ceranae infecting eastern honeybee workers
}

Yuanchan Fan ${ }^{1}$, Jie Wang ${ }^{1}$, Kejun $\mathrm{Yu}^{1}$, Wende Zhang ${ }^{1}$, Zongbin Cai ${ }^{1}$, Minghui Sun ${ }^{1}$, Ying Hu ${ }^{1}$, Xiao Zhao ${ }^{1}$, Cuiling Xiong ${ }^{1}$, Qingsheng Niu ${ }^{3}$, Dafu Chen ${ }^{1,2, *}$, Rui Guo ${ }^{1,2, *}$

${ }^{1}$ College of Animal Sciences (College of Bee Science), Fujian Agriculture and Forestry University, Fuzhou, 350002, Fujian, China

${ }^{2}$ Apitherapy Research Institute, Fujian Agriculture and Forestry University, Fuzhou, 350002, Fujian, China

${ }^{3}$ Jilin Province Institute of Apicultural Science, Jiling 132000

*Correspondence: ruiguo@fafu.edu.cn, dfchen826@fafu.edu.cn; Tel./Fax:+86-0591-8764-0197

\begin{abstract}
Apis cerana is the original host for Nosema ceranae, a widespread fungal parasite resulting in bee nosemosis, which leads to severe losses for apiculture industry throughout the world. However, knowledge of $N$. ceranae infecting eastern honeybees is extremely limited. Currently, the mechanism underlying N. ceranae infection is still largely unknown. Based on our previously gained high-quality transcriptome datasets, comparative transcriptomic investigation was conducted in this work, with a focus on virulence factor-associated differentially expressed genes (DEGs). Microscopic observation showed that A. c. cerana workers' midguts were effectively infected after inoculation with clean spores of $N$. ceranae. Totally, 1411, 604, and 38 DEGs were identified from NcCK vs. NcT1, NcCK vs. NcT2 and NcT1 vs. NcT2 comparison groups. Venn analysis showed that ten up-regulated genes and nine down-regulated ones were shared by aforementioned comparison groups. GO category indicated these DEGs were involved in a series of functional terms relevant to biological process, cellular component, and molecular function, such as metabolic process, cell part, and catalytic activity. Additionally, KEGG pathway analysis suggested that the DEGs were engaged in an array of pathways of great importance, such as metabolic pathway, glycolysis, and biosynthesis of secondary metabolites. Further,
\end{abstract}


expression clustering analysis demonstrated that majority of genes encoding virulence factors such as ricin B lectins and polar tube proteins displayed apparent up-regulation, whereas a few virulence factor-associated genes such as hexokinase gene and 6-phosphofructokinase gene presented downregulation during the fungal infection. Finally, the expression trend of 14 DEGs was confirmed by RTqPCR, validating the reliability of our transcriptome datasets. These results together demonstrated that an overall alteration of the transcriptome of $N$. ceranae occurred during the infection of $A$. c. ceranae workers, and most of virulence factor-related genes were induced to activation to promote the fungal invasion. Our findings not only lay a foundation for clarifying the molecular mechanism underlying N. ceranae infection of eastern honeybee workers, but also shed light on developing novel targets for microsporidiosis control.

Keywords: Honeybee; Apis cerana cerana; Nosema ceranae; microsporidian; Transcriptome; Differentially expressed gene; Infection mechanism

\section{Introduction}

Nosema ceranae is an obligate unicellular fungal parasite that specifically infects bee midgut epithelial cells. $N$. ceranae had been first identified in eastern honeybee (Apis cerana) by Fries et al. [1], thereafter it swiftly spread to western honeybee (Apis mellifera) colonies reared in Europe and Taiwan province, China [2]. Currently, N. ceranae could be detected in colonies all over the world. $N$. ceranae infestation results in a battery negative impact on bee host, such as shortened life span, energy stress, immunosuppression, cell apoptosis inhibition $[3,4,5,6]$, earlier foraging activity, and impaired navigation and cognitive ability [7,8]. A close connection between $N$. ceranae and colony collapse disorder (CCD) had been suggested by several studies $[9,10]$.

$N$. ceranae exists outside the host cell only as dormant spores. After ingestion by the bee host, the inside polar tube is rapidly extruded to pierce the cell membrane followed by injection of the infective 
sporoplasm via hollow polar tube $[1,11,12]$. The intracellular life cycle of $N$. ceranae can be divided into two phases including the proliferative phase (merogony) and the sporogonic phase (sporogony), and ends with the formation of spores. Nevertheless, it's hard to completely isolate $N$. ceranae at above-mentioned two different phases, which is a key factor limiting further study on the fungal parasite during the infection process. Advances in next-generation sequencing technology have allowed a deeper understanding of host response and parasite/pathogen infection. Previously, several studies were conducted to investigate responses of western honeybee workers to microsporidian infestation, Badaoui et al. analyzed the gene expression of $A$. $m$. ligustica workers at 5, 10, and 15 days post $N$. ceranae infection by RNA-seq technology, and found that the expression of genes encoding host antimicrobial peptides, cuticle proteins, and odor binding proteins were down-regulated, resulting in the decline of immune function of the host, so as to promote the survival and propagation of $N$. ceranae at the colony level [13]; based on fluorescence in situ hybridization (FISH) and immunostaining experiments, Panek et al. investigated the impact of $N$. ceranae on $A$. m. ligustica workers epithelium renewal by following the mitotic index of midgut stem cells during a 22-day $N$. ceranae infection, the results showed that $N$. ceranae can negatively alter the gut epithelium renewal rate and disrupt some signaling pathways involved in the gut homeostasis [14]. Transcriptome analysis of the intestinal tract of $A$. c. cerana exposed to $N$. ceranae demonstrated that microsporidian infection inhibited genes relevant to homeostasis and renewal in wnt signaling pathway [15]. Comparatively, omics study on the $N$. ceranae infecting bee hosts is very limited, and the underlying mechanism of $N$. ceranae infection is still vague.

In our previous work, we performed RNA sequencing of clean spores of $N$. ceranae, followed by transcriptome-wide identification of ncRNAs such as miRNAs, lncRNAs, and circRNAs in N. ceranae 
spores $[16,17,18]$. Recently, we conducted deep sequencing of the midguts of $A$. c. cerana workers at 7 and 10 days post inoculation (dpi) with $N$. ceranae spores, and deciphered the host cellular and humoral responses to fungal infection [19]; in addition, we analyzed the expression profile of highly expressed genes (HEGs) and discussed their potential roles in N. ceranae infestation [20]. In view of that transcriptomic study on $N$. ceranae during the infection process is still very lagging, in the present study, based on the obtained transcriptome datasets, we first filtered out the parasite-derived data and then performed comparative investigation combined with transcriptome data derived from N. ceranae spores, followed by deep investigation of the dynamics of genes in $N$. ceranae as well as virulence factor-associated pathways and genes. To the best of our knowledge, this is the first report of omics study on $N$. ceranae invading eastern honeybee.

\section{Results}

\subsection{Verification of infection of $A$. c. cerana worker by $N$. ceranae}

Under optical microscope, oval and highly refractive dispersed spores were observed (Figure 1A). Further, AGE indicated that expected fragment (approximately $76 \mathrm{bp}$ ) was amplified from the purified spores with specific primers for $N$. ceranae, while no signal band was detected using specific primers for $N$. apis (Figure 1B). These results verified that the purified spores were indeed $N$. ceranae spores.

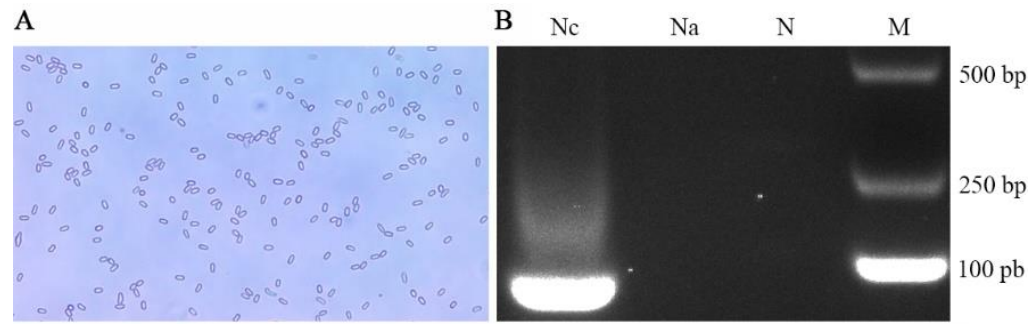

Figure1. Microscopic detection and PCR validation of $N$. ceranae spores. (A) Microscopic detection (400 times amplification). (B) AGE for PCR amplified fragments, Lane Nc: Specific 
primers for $N$. ceranae, Lane Na: Specific primers for $N$. apis, Lane N: Sterile water (Negative control), Lane M: DNA marker.

Based on microscopic observation of paraffin sections, it's found that there were a number of $N$. ceranae spores in the A. c. cerana worker's midgut epithelial cells at 11 dpi with N. ceranae (Figure 2A-B), whereas no fungal spores can be detected in the worker's midgut epithelial cells at 11 dpi without $N$. ceranae (Figure 2C-D). In addition, the structure of midgut epithelial cells of $N$. ceranaeinfected worker was fragmentary and the nucleic acid substances were dispersed and unclear (Figure 2A-B), while that of un-infected worker was intact and the deeply colored cell nucleus were visible (Figure 2C-D). The results together suggested that the A. c. cerana workers were infected by $N$. ceranae and the host midgut epithelial cell structure was destructed by the fungal infection.

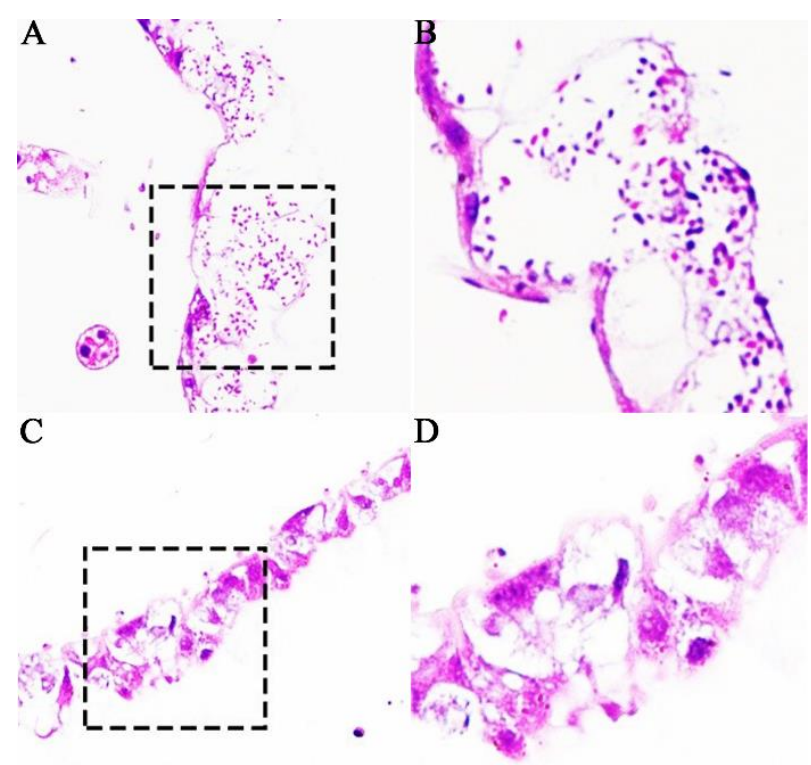

Figure2. Microscopic observation of paraffin sections of $N$. ceranae-inoculated and uninoculated $A$. c. cerana workers' midguts. (A) Worker's midgut at 11 dpi without $N$. ceranae under 200 times amplification; (B) Worker's midgut at 11 dpi without $N$. ceranae under 400 times amplification. Black dashed box shows the region for observation under 400 times amplification. 


\subsection{Differential gene expression profile of $N$. ceranae infecting $A$. c. cerana workers}

Totally, 1411, 604, and 38 DEGs were identified in NcCK vs. NcT1, NcCK vs. NcT2, and NcT1 vs. NcT2 comparison groups, respectively. The numbers of up-regulated genes were 711, 240, and 17, while those of down-regulated genes were 700, 360, and 21, respectively (Figure 3A). Additionally, Venn analysis showed that there were 10 and nine shared up- and down-regulated genes in the aforementioned three comparison groups, whereas the numbers of unique up-regulated (downregulated) genes were 417 (354), two (20), and five (10), respectively (Figure 3B).

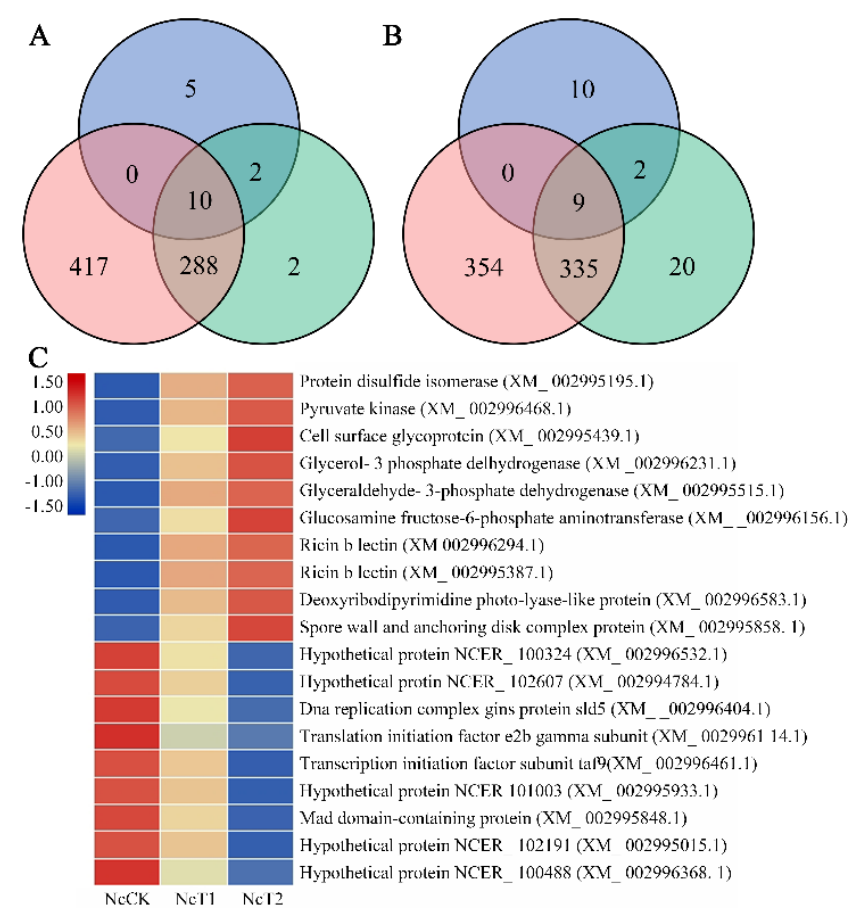

Figure 3. Analysis of DEGs in N. ceranae. (A) Venn analysis of up-regulated genes in NcCK $v s$. NcT1, NcCK vs. NcT2, and NcT1 vs. NcT2 comparison groups. (B) Venn analysis of downregulated genes in NcCK vs. NcT1, NcCK vs. NcT2, and NcT1 vs. NcT2 comparison groups. (C) Heatmap of common DEGs in NcCK, NcT1, and NcT2 groups.

\subsection{Function and pathway annotation of DEGs in $N$. ceranae infesting $A$. c. cerana workers}

GO term analysis suggested that the DEGs in NcCK vs. NcT1 comparison group were engaged in 638 biological process-associated functional terms such as metabolic process, single-organism process, 
and cellular process; 377 cellular component-associated terms such as cell, cell part, and organelle; and 424 molecular function-associated terms such as catalytic activity, transport, and binding (Figure 4A). In NcCK vs. NcT2 comparison group, the DEGs could annotate to 22 GO terms, including 291 biological process-related items such as metabolic process and single-organism, 160 cellular component-related terms such as cell and cell part, and 237 molecular function-related items such as catalytic activity and nucleic acid binding transcription factor activity (Figure 4B). The DEGs in NcT1 vs. NcT2 comparison group were involved in seven functional terms such as metabolic process, cell, and catalytic activity (Figure 4C).
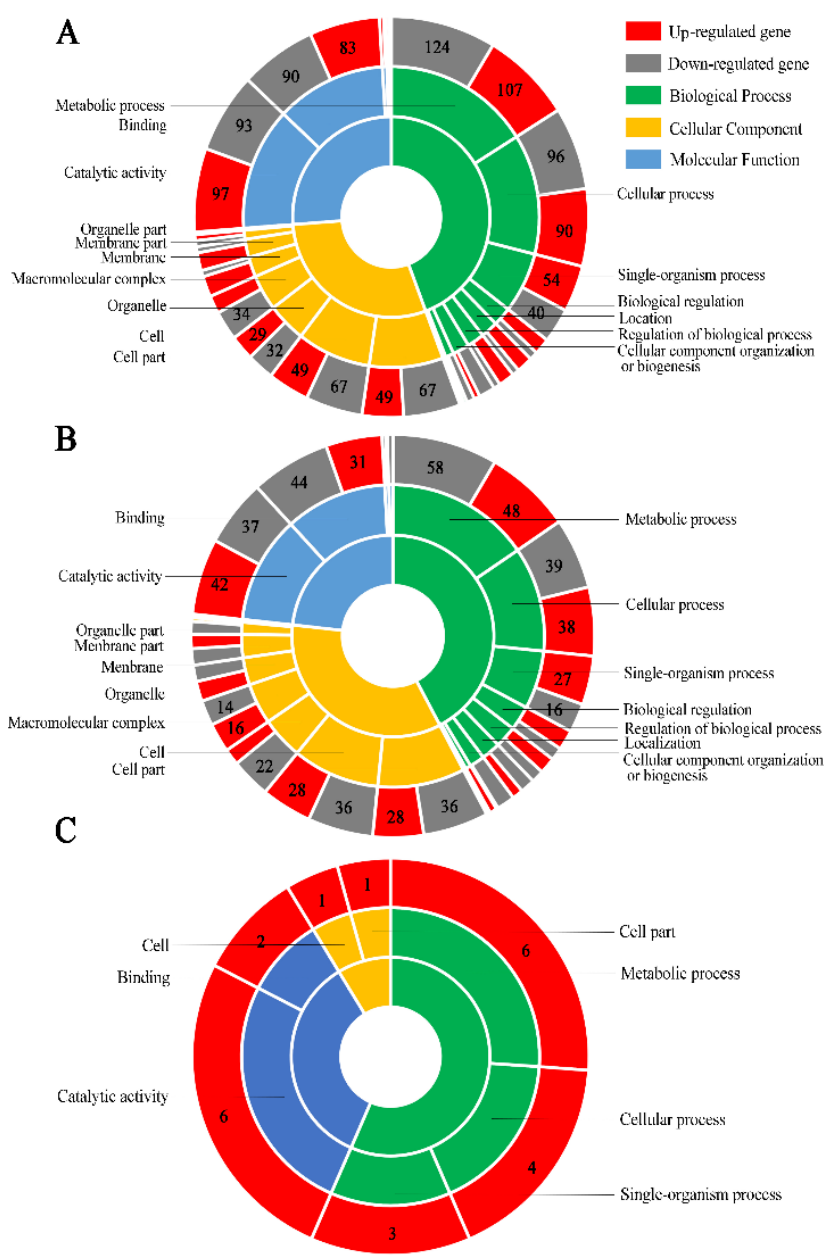

Figure 4. GO category of DEGs. (A) DEGs in NcCK vs. NcT1. (B) DEGs in NcCK vs. NcT2; (C) DEGs in NcT1 vs. NcT2. 
In addition, KEGG pathway analysis indicated that the DEGs in NcCK vs. NcT1 comparison group were relevant to 241 pathways, among these the most abundant groups were metabolic pathway, biosynthesis of secondary metabolites, ribosome, ribosome biogenesis in eukaryotes, and biosynthesis of antibiotics (Figure 5A). In NcCK vs. NcT2 comparison group, the DEGs were relative to 201 pathways including metabolic pathway, biosynthesis of secondary metabolites, ribosome, ribosome biogenesis in eukaryotes, and biosynthesis of antibiotics (Figure 5B). The DEGs in NcT1 vs. NcT2 comparison group were associated with 32 pathways such as metabolic pathway, biosynthesis of antibiotics, biosynthesis of secondary metabolites, glycolysis/gluconeogenesis, and microbial metabolism in diverse environments (Figure 5C). 

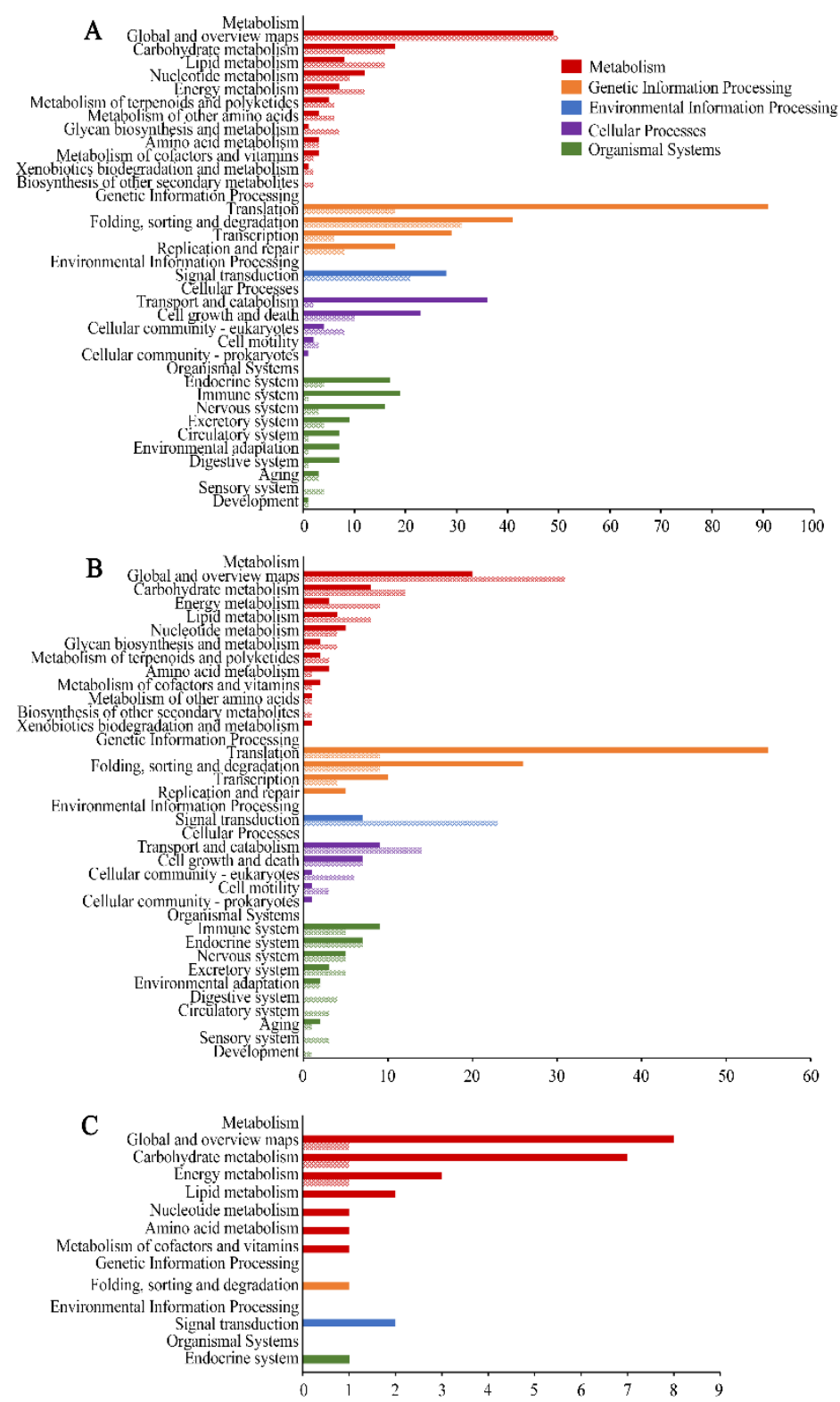

Figure 5. Pathways annotated by DEGs. (A) DEGs in NcCK vs. NcT1. (B) DEGs in NcCK vs. NcT2. (C): DEGs in NcT1 vs. NcT2.

\subsection{Virulence factor-associated DEGs in $N$. ceranae invading A. c. cerana workers}

Further investigation was conducted to explore virulence factor-associated DEGs in abovementioned comparison groups, a total of 20 DEGs were identified, including six spore wall protein coding genes, three ricin B lectin protein coding genes, three ATP/ADP translocase protein coding genes, two polar tube protein coding genes, two $\mathrm{ABC}$ transporter protein coding genes, one chitin 
synthase protein coding gene, one 6-phosphofructokinase protein coding genes, one hexokinase protein coding gene, and one pyruvate kinase protein coding gene. Moreover, the expression clustering showed that majority of virulence factor-encoding genes were induced to activation during the infection process, whereas a few were suppressed to a large externt (Figure 6).

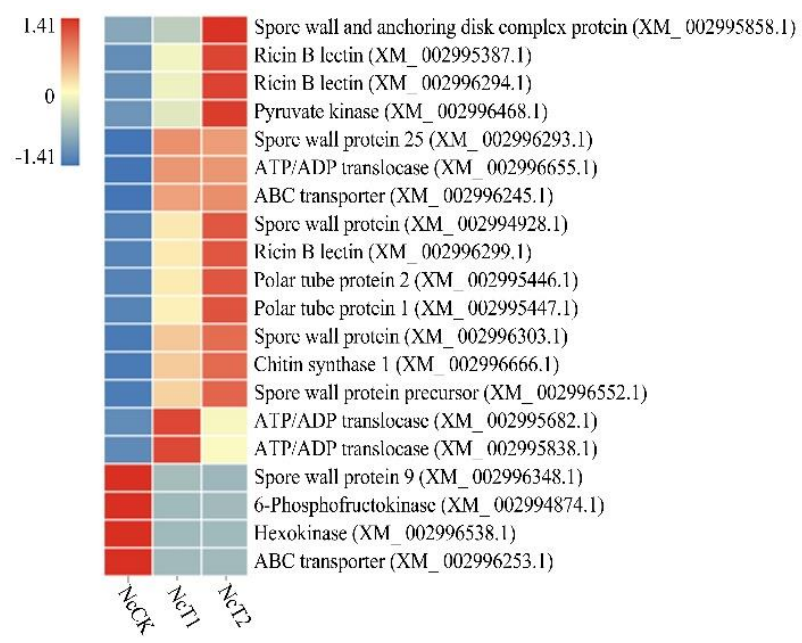

Figure 6. Heatmap of virulence factor-associated DEGs shared by NcCK, NcT1, and NcT2 groups.

\subsection{Verification of DEGs via RT-qPCR}

Fifteen DEGs were randomly selected for RT-qPCR validation, the result suggested that the expression trend of 14 were consistent with those in transcriptome data (Figure 7A-B), confirming the reliability of the sequencing data used in this current work. 

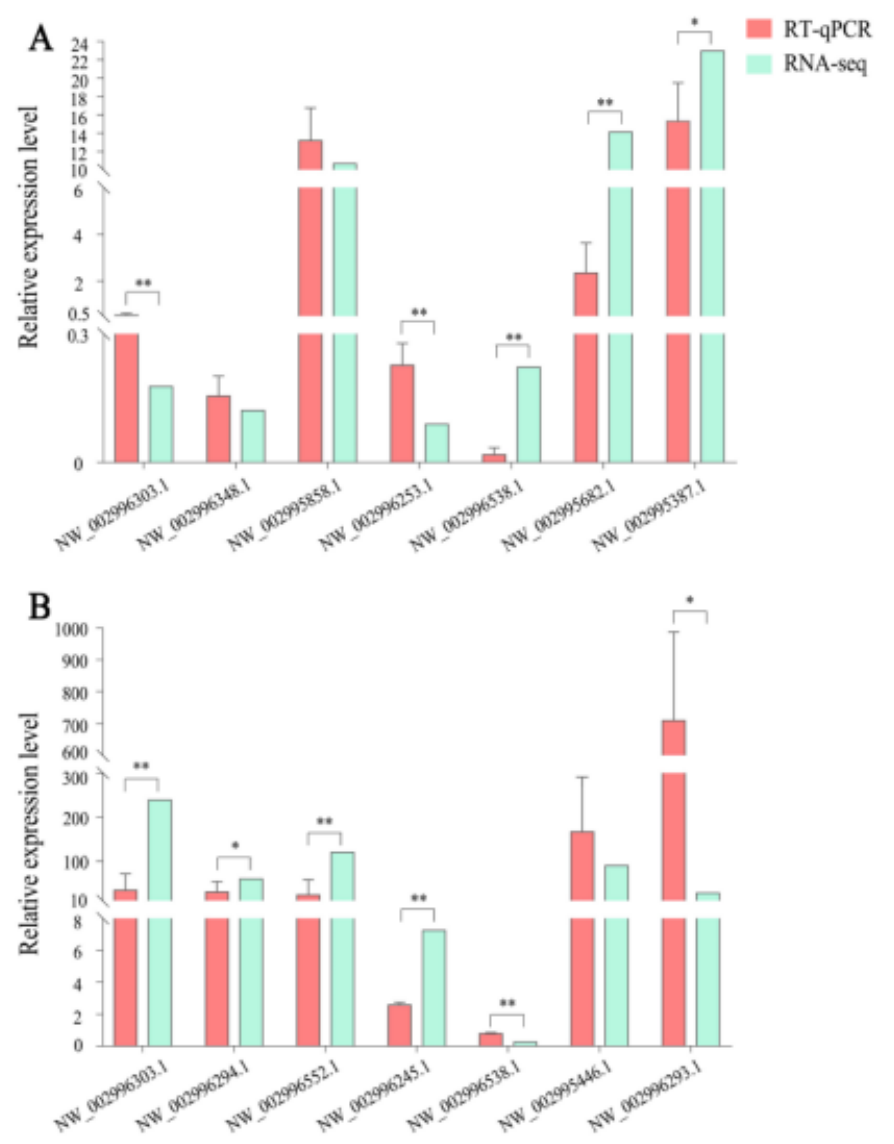

Figure 7. RT-qPCR verification of DEGs in N. ceranae infecting A. c. cerana workers. (A)

DEGs in NcCK vs. NcT1. (B) DEGs in NcCK vs. NcT2. Error bars represent the variance of RT-qPCR results of each DEG. Bars with asterisk symbol indicate statistical differences $(\mathrm{P}<$ 0.05).

\section{Discussion}

Currently, the mechanism regulating $N$. ceranae infestation remains to be clarified. Here, based on our previously obtained transcriptome data from $N$. ceranae-inoculated and un-inoculated $A$. $c$. cerana workers' midguts at $7 \mathrm{dpi}$ and $10 \mathrm{dpi}$, the mixed transcriptome data were first filtered to gain those $N$. ceranae-derived data, and then used for comparative analysis in conjunction with transcriptome data from $N$. ceranae spores. Following the similar technique protocol, our them previously investigated transcriptomic dynamics of $N$. ceranae invading A. m. ligustica workers and Ascosphaera apis infecting A. m. ligustica or A. c. cerana larvae [30,31,33-35], and the protocol was 
verified to be feasible and reliable, offering a reference for investigating pathogens or endoparasites during the infection process, especially those hardly isolated from hosts under current technical conditions.

In NcCK vs. NcT1 and NcCK vs. NcT2 comparison groups, 1411 and 604 DEGs were respectively identified, including 657 and 240 up-regulated genes as well as 700 and 364 down-regulated ones. This indictive of the overall alteration of genes in $N$. ceranae during the infection process. GO term analysis showed that the aforementioned DEGs were involved in 24 and 22 functional terms associated with biological processes such as metabolic process and cellular process, cellular components such as cell and organelle, and molecular function such as catalytic activity and transport. In addition, these DEGs were annotated to 241 and 201 pathways, respectively, including material metabolism-related pathways such as Corban metabolism and energy mechanism-related pathways such as glycolysis/gluconeogenesis. The response of fungal cells to external signals is regulated by mitogenactivated protein kinase (MAPK), which phosphorylates many downstream proteins and further alter gene expression, followed by participation in various process such as proliferation, differentiation, and apoptosis [36]. In this current work, three and two DEGs in NcCK vs. NcT1 and NcCK vs. NcT2 were found to enrich in MAPK signaling pathway, among which XM_002995908.1 $(\log 2 \mathrm{FC}=4.44,4.93)$ and XM_002996683.1 $(\log 2 \mathrm{FC}=4.63,4.51)$ were shared by the both comparison groups, while there was a unique DEG (XM_002996282.1, $\log 2 \mathrm{FC}=-1.01)$ in NcCK vs. NcT1. This indicated that MAPK signaling pathway was significantly activated during $N$. ceranae infection and used by the fungal parasite to response to the internal environment of epithelial cells in A. c. cerana workers' midguts. Previously, we conducted transcriptomic investigation of A. m. ligustica workers' midguts responding to $N$. ceranae invasion, and detected that seven (GenBank accession no. 9423510, 424610, 9424462, 9422987, 9422306, 9411598 and 9424007) DEGs in midguts at 7 dpi and 10 dpi were enriched in MAPK signaling pathway [31], which suggested that the MAPK pathway regulated A. m. ligustica immune response to $N$. ceranae. These results together demonstrated that MAPK signaling pathway 
was likely to play a vital part in $N$. ceranae infection, but there were differences of this signaling pathway in N. ceranae when infecting different bee species.

Ricin, a kind of toxic heterodimer protein in castor seed, has a ricin chain B (RTB) that connects a ricin chain A (RTA) via a disulfide bond [37]. Lectins are proteins that recognize glycan ligand. As virulence factors of many pathogens, lectins exert function by mediating cell adhesion, innate immune defense, and pathogen infection [38]. The binding of ricin B lectin (RBL) contributes to microbial infection and promotes pathogen attachment or entry into host cells. NBRBL3, a lectin protein of Nosema bombyx, was confirmed to be secreted from the spore during fungal proliferation and then mediate host cell recognition and adhesion [38]. In this research, three ricin B lectin-associated DEGs were observed in both $\mathrm{NcCK}$ vs. $\mathrm{NcT} 1$ and $\mathrm{NcCK}$ vs. NcT2 comparison groups, including XM_002995387.1 $(\log 2 \mathrm{FC}=4.52, \quad 5.67), \quad$ XM_002996294.1 $\quad(\log 2 \mathrm{FC}=4.66, \quad 5.88), \quad$ and XM_002996299.1( $\log 2 \mathrm{FC}=2.31,2.96)$, and all of these were apparently up-regulated, implying that $N$. ceranae may synthesize and secrete ricin B lectin to increase the adhesion between spores and host cells to promote fungal proliferation [31].

There is a hard spore wall outside the microsporidia, which is composed of high electron density protein outer spore wall layer, low electron density chitin inner spore wall layer, and fibrous plasma membrane. Spore wall protein (SWP) is proved to be a crucial virulence factor of microsporidia, which can recognize and adhere to host cells during microsporidian infection [39]. The spore wall protein SWP25 of Nosema bombyx was located in the endospore layer with a signal peptide, which was suggested to be implicated in chitin interaction of endospore powder and the construction of spore wall via the heparin binding motif (HBM) [40]. Here, one SWP25-encoding gene (XM_002996293.1) showed an up-regulation trend in host midguts at $7 \mathrm{dpi}(\log 2 \mathrm{FC}=4.82)$ and 10 dpi $(\log 2 \mathrm{FC}=4.77)$, suggestive of the participation of SWP25 in N. ceranae infection of A. c. cerana workers. Yang et al. analyzed the interaction between Nosema bombycis SWP9 (NbSWP9) with polar tube proteins PTP1 and PTP2 by molecular methods such as co-immunoprecipitation and yeast two-hybrid assay, and 
found that NbSWP9 was mainly distributed in the polar tubes [41]. In this work, one gene encoding SWP9 (XM_002996348.1) was down-regulated in workers' midguts at both 7 dpi $(\log 2 \mathrm{FC}=-3.04)$ and $10 \mathrm{dpi}(\log 2 \mathrm{FC}=-3.40)$, indicating that $A$. c. cerana workers may inhibit the interaction between SWP9 with PTPs via host-parasite interaction. However, additional work is required to decipher the underlying mechanism. In addition, another two SWP encoding genes (XM_002996303.1, $\left.\log 2 \mathrm{FC}=7.45,7.90 ; \mathrm{XM} \_002994928.1, \log 2 \mathrm{FC}=5.08,5.82\right)$, one SWP precursor encoding gene (XM_002996552.1, $\log 2 \mathrm{FC}=6.36,6.90)$, and one spore wall and anchoring disk complex protein encoding gene (XM_002995858.1, $\log 2 \mathrm{FC}=3.41,5.80)$ were identified and all of them were observed to up-regulate in host midguts at both $7 \mathrm{dpi}$ and $10 \mathrm{dpi}$, which demonstrated that these SWP-associated genes were activated to increase the synthesis of corresponding SWPs, further enhancing the N. ceranae invasion.

For microsporidia, the infective protoplasm is transferred from the spore to host cell through the polar tube, further starting the proliferation process. Therefore, the polar tube protein is also considered as a virulence factor of importance. Yang et al. discovered that the polar tube and spore wall of microsporidia are the main components of mature spores adhering to and infecting the host cells [42]. Long et al. performed lectin blotting and $\beta$-elimination reaction of NbPTP1 in N. bombycis, the results showed that NbPTP1 had O-glycosylation modification characteristics, which was conducive to adhesion, infection, and maintenance of the stability of the polar tube. Moreover, the PTP1 protein sequence contains high proline, which can improve the elasticity of the polar tube [43]. Here, two PTP1 encoding genes shared by the aforementioned two comparison groups were found to be upregulated, including XM_002995447.1 $(\log 2 \mathrm{FC}=6.01,6.84)$ and XM_002995446.1 $(\log 2 \mathrm{FC}=5.71$, 6.48), indicating that the tubulin was engaged in the infection process of $N$. ceranae and played a role in the maintenance of the stability of polar tube.

Chitin is a major component of the spore wall of $N$. ceranae. Chitin synthase is an important enzyme implicated in chitin biosynthesis. Cabib et al. discovered that chitin synthase 1 and chitin 
synthase 2 exerted synergistic function in chitin repair in yeast, and jointly participated in the cell wall construction [44]. In the present study, the chitin synthase 1 encoding gene (XM_002996666.1) was up-regulated in both $\mathrm{NcCK} v s . \mathrm{NcT} 1(\log 2 \mathrm{FC}=4.07)$ and $\mathrm{NcCK} v s . \mathrm{NcT} 2(\log 2 \mathrm{FC}=4.52)$ comparison groups, implying that it played a part in the construction and repair of spore wall to assist the $N$. ceranae infection. However, we did not detect the differential expression of chitin synthase gene in $N$. ceranae infecting the A. m. ligustica workers. It's speculated that chitin synthesis gene was a special virulence factor for N. ceranae, playing different roles when infecting different bee species.

Owing to the specific life cycle, microsporidia lost the typical mitochondria during the long-term evolution, which was replaced by mitosis, an organelle that produces a small amount of ATP via glycolysis to meet the basic physiological needs [11]. Accordingly, microsporidia highly depend on stealing energy from the host cells, and glycolysis is the main manner for $N$. ceranae to produce ATP at a pretty low efficiency [12]. Huang et al. conducted transcriptomic analysis of the $N$. ceranae during the 6 days infection cycle, and detected that the genes encoding three rate-limiting enzymes involving catalyzing glycolysis pathway continued to up-regulate, including hexokinase, pyruvate kinase, and phosphofructokinase; and at the end of infection ( $6 \mathrm{dpi})$, the gene expression levels of ATP/ADP translocase gene, ABC transporter gene, and catalytic glycolysis encoding gene were plunged [45]. Hexokinase catalyzes glucose phosphorylation to form glucose-6-phosphate (g-6-p), and the expression of hexokinase is regulated by ADP. Pyruvate kinase can catalyze the conversion of phosphoenolpyruvate to pyruvate and further enhance the production of ATP. Fructose-6-phosphate (f-6-p) is hydrolyzed by 6-phosphofructokinase, glucose-1 (F-1) and 6-diphosphate (6-2p) were inhibited by ATP with high concentration [56-48]. Noticeably, the pyruvate kinase encoding gene XM_002996468.1 $(\log 2 \mathrm{FC}=2.81,3.92)$ and hexokinase encoding gene XM_002995838.1 $(\log 2 \mathrm{FC}=$ 3.78, 3.23) were up-regulated in $\mathrm{NcCK} v s . \mathrm{NcT} 1$ and $\mathrm{NcCK} v s . \mathrm{NcT} 2$, which suggested that activation of pyruvate kinase and hexokinase was a strategy of $N$. ceranae during the infection of $A$. c cerana workers. However, the 6-phosphofructokinase encoding gene XM_002994874.1 ( $\log 2 \mathrm{FC}=-4.72$, - 
4.03) was down-regulated, which was inconsistent with previous study [45]. We inferred that it might be suppressed by ATP with high concentration. The underlying mechanism needs to be further explored.

$\mathrm{ABC}$ transporters represent the largest family of transmembrane proteinsm which contribute to transportation of ATP. Most of ABC transporters rely on ATP binding and hydrolysis to transport amino acids, lipids, sugars, peptides, ions, and other substrates from the cell fluid to intracellular or extracellular region [49]. He et al. identified 234 ABC transporters from 18 microsporidium genomes and divided them into five subfamilies: ABCBs, ABCCs, ABCEs, ABCFs, and ABCGs. Two subfamilies of $\mathrm{ABCBs}$ and $\mathrm{ABCDs}$ were lost in microsporidian genome. On the basis of $\mathrm{qPCR}$, Western blotting, and RNAi, He et al. revealed that NoboABCG1.1 regulated the fungal reproduction and promoted the proliferation of $N$. bombycis [50]. Here, the expression trend of two ABC transporterencoding genes was altered in the above-mentioned two comparison groups; XM_002996245.1 $(\log 2 \mathrm{FC}=2.52,2.86)$ was up-regulated, while XM_002996253.1 $(\log 2 \mathrm{FC}=-3.49,-3.68)$ was downregulated. This indicated that $\mathrm{ABC}$ transporters may play an essential role in the transmembrane transport of materials and energy during the $N$. ceranae infection, and different $\mathrm{ABC}$ transporters encoding genes may have different roles in this process.

ATP/ADP translocase transports ATP synthesized in mitochondrial matrix to cytoplasm for cell utilization in eukaryotes. $N$. ceranae needs to steal ATP from host cells by ATP/ADP translocase to meet its growth and development needs. Paldi et al. found four ATP/ADP translocases in N. ceranae infecting A. mellifera workers based on genomic sequencing and investigation, and concluded that ATP/ADP translocases may play a role in transporting ATP from hose cells to the mitosis in N. ceranae [51]. In the present study, the up-regulation of two ATP/ADP translocase genes was detected in NcCK vs. NcT1 and NcCK vs. NcT2, including XM_002995682.1( $\log 2 \mathrm{FC}=3.82, \quad 3.33)$, XM_002996655.1 $(\log 2 \mathrm{FC}=2.54,2.54)$; whereas another gene XM_002996538.1 $(\log 2 \mathrm{FC}=-2.08$, 2.08) was down-regulated, indicative of the complex interaction between microsporidian and host. The 
result supported the vital function of ATP/ADP transporters in energy acquisition of $N$. ceranae invading A. c. cerana workers.

\section{Materials and Methods}

\subsection{Fungal spore and honeybee}

Clean spores of $\mathrm{N}$. ceranae were previously purified using Percoll discontinuous gradient centrifugation protocol and preserved in Honeybee Protection Laboratory [18], College of Animal Sciences (College of Bee Science). A. c. cerana workers were selected from three colonies located in the teaching apiary of the College of Animal Sciences (College of Bee Science) in Fujian Agriculture and Forestry University. No Varroa mite was observed during the whole study. The disappearance of seven common bee viruses (KBV, IAPV, ABPV, DWV, SBV, BQCV, and CBPV) and two bee microsporidia (Nosema apis and N. ceranae) in the newly emergent A. c. cerana workers was verified with RT-PCR assay [21].

\subsection{Microscopic observation and PCR validation of $N$. ceranae spores}

The prepared spores of $\mathrm{N}$. ceranae was subjected to microscopic observation using an optical microscope (SIOM, Shanghai, China). Further, total DNA of spores were isolated and used as templates for reverse transcription; the resulting cDNA was then used as templates for PCR amplification with previously described specific primers for N. ceranae and N. apis [22-23]; the amplified products were detected by $1.5 \%$ agarose gel electrophoresis (AGE). Sterile water was set as a negative control.

\subsection{Preparation and detection of paraffin section of honeybee midgut tissue}

One-day-old workers of A. c. cerana in N. ceranae-inoculated groups were each artificially fed with 5 ul 50\% (w/v) sucrose solution containing $1 \times 106$ spores; while 1-old-day workers in uninoculated groups were each fed with $5 \mu \mathrm{L}$ of $50 \%(\mathrm{w} / \mathrm{v})$ sucrose solution without spores. At 11 dpi 
with N. ceranae spores, the midgut tissues in the N. ceranae-inoculated and un-inoculated groups were respectively harvested and fixed with $4 \%$ paraformaldehyde. According to our previously described protocol [21], on the basis of a microtome (Leica, Nussloch, Germany) and an embedding center (Junjie, Wuhan, China), paraffin sections were prepared and then stained with hematoxylin eosin (HE) stain by Shanghai Sangon Biological Engineering Co. Ltd, followed by detection utilizing an optical microscope with digital camera (SOPTOP, Shanghai, China).

\subsection{Transcriptome data source}

Midgut tissues of A. c. cerana workers at 7 dpi and 10 dpi with $N$. ceranae spores and corresponding un-inoculated worker's midgut tissues were previously prepared and sequenced on Illumina HiSeqTM 4000 platform with a stand-specific cDNA library-based strategy [17]. The raw datasets had been deposited in the National Biotechnology Information Center (NCBI) SRA database (https://www.ncbi.nlm.nih.gov/sra) under BioProject number: PRJNA395264. Quality control of the produced raw reads were previously conducted according to the method described by Chen et al $[17,24]$. Briefly, raw reads containing adapters, more than $10 \%$ of unknown nucleotides $(\mathrm{N})$, and more than $50 \%$ of low quality (q value $\leq 20$ ) bases were removed to gain high-quality clean reads, which were then mapped to the ribosome RNA (rRNA) database (www.arb-silva.de/) (20 March 2021) using Bowtie2 software [24]. The result indicated that 174, 700, 032, 205, 297, 946, 124, 216, 829, and 99, 030, 788 raw reads were generated, and after quality control, 171, 868, 061, 200, 570, 776, 121, 949, 977, and 97, 432, 267 clean reads were gained, with Q20 of 94.96\%, 94.58\%, 94.60\%, and 94.91\% respectively [25]. Hence, the high-quality RNA-seq data can be used for transcriptomic analyses in the present study. In another work, we conducted deep sequencing of clean spores of N. ceranae (NcCK) using Illumina HiSeq-based RNA-seq [18]. A total of 416, 156, 600 raw reads were produced, and after quality control, 210, 824, 312 clean reads were obtained, with average Q30 of 92.63\%, which suggested that the high-quality transcriptome data can be used in this study. The relevant raw data had 
been deposited in NCBI SRA database (https://www.ncbi.nlm.nih.gov/sra) under BioProject number: PRJNA562784.I

\subsection{Identification and analysis of DEGs in N. ceranae}

Following the standard of $\mathrm{P}$ value $\leq 0.05$ and $\mid \log 2$ (Fold change) $\mid \geq 1$, the DEGs in NcCK vs. NcT1 and NcCK vs. NcT2 comparison groups were screened by edgeR software [26]. Venn analysis of up- and down-regulated genes in these comparison groups and expression cluster analysis were carried out based on OmicShare platform (https://www.omicshare.com/). GO (Gene Ontology) categorization of DEGs were carried out using WEGO software [27]. Blastall tool was employed to conduct pathway analysis by comparing DEGs against KEGG (Kyoto Encyclopedia of Genes and Genomes) database (https://www.kegg.jp/) [28].

\subsection{Investigation of virulence factor-associated DEGs}

On the basis of associated documentations with N. ceranae and findings from our previous studies on N. ceranae [16-20, 23, 29-31], virulence factors such as spore wall protein, ricin B lectin, chitinase, polar tube protein, phosphofructokinase, ATP/ADP translocase, ABC transporter, hexokinase, and pyruvate kinase and associated DEGs were selected for further investigation. Expression clustering analysis of aforementioned DEGs was performed utilizing OmicShare platform.

\subsection{RT-qPCR validation of DEGs}

Fifteen DEGs were randomly selected from NcCK vs. NcT1 (XM_002994928.1, XM_002996348.1,XM_002996299.1, XM_002996655.1, XM_002996468.1 and XM_002996253.1), NcCK vs. NcT2 (XM_002996303.1, XM_002996294.1, XM_002995682.1, XM_002996538.1, XM_002996655.1 and XM_002996253.1), and NcT1 vs. NcT2 (XM_002996294.1 and XM_002996468.1) comparison groups and subjected to RT-qPCR validation. actin gene (gene6001) was used as an internal reference. Specific forward and reverse primers for these DEGs and actin were 
designed with primer premier 5 (Table SI). Total RNA of N. ceranae spores and N. ceranae-inoculated workers' midguts at 7 dpi and 10 dpi were respectively isolated using RNA Extraction Kit (TaKaRa company, Dalian, China). cDNA was synthesized through reverse transcription with oligo dT primer and used as templates for qPCR assay, which was carried out on a QuanStudio RealTime PCR System (ThemoFisher, Walthem, MA, USA). The qPCR reaction was conducted according to the instructions of SYBR Green Dye Kit (Vazyme company, Shanghai, China). Cycling parameters were as follows: $95{ }^{\circ} \mathrm{C}$ for $1 \mathrm{~min}$, followed by 40 cycles at $95^{\circ} \mathrm{C}$ for $15 \mathrm{~s}, 55^{\circ} \mathrm{C}$ for $30 \mathrm{~s}$, and $72{ }^{\circ} \mathrm{C}$ for $45 \mathrm{~s}$. The relative gene expression was calculated based on $2-\Delta \Delta \mathrm{Ct}$ method [32]. The experiment was performed three times utilizing three independent biological samples.

\subsection{Statistical analysis}

Statistical analyses were conducted with SPSS software (IBM, Amunque, NY, USA) and GraphPad Prism 7.0 software (GraphPad, San Diego, California, USA). Data were presented as mean \pm standard deviation (SD). Statistics analysis was performed using Student's t-test and one-way ANOVA. Additionally, significant $(p<0.05)$ GO terms and KEGG pathways were filtered by performing Fisher's exact test with R software 3.3.1.

\section{Conclusions}

In summary, our results demonstrated that the overall transcriptome dynamics of N. ceranae was altered during the infection of A. c. cerana workers, a number of virulence factor-associated genes were induced to activation to facilitate the fungal infestation, but some other genes encoding virulence factors were suppressed via host-microsporidian interaction. Findings in this current work not only provide a basis for clarifying the mechanism underlying the N. ceranae infection of A. c cerana workers, but also shed light on the development of novel strategy for nosemosis control.

Supplementary Materials: Table S1 Primers for RT-qPCR validation performed in this study. 
Author Contributions: DFC and RG designed this study. RG, YCF, JW, KJY, WDZ, ZBC, MHS, YH, XZ and CLX performed bioinformatic analysis and molecular experiment. DFC, QSN and RG supervised the work and contributed to preparation of the manuscript.

Funding: This work was founded by the National Natural Science Foundation of China (32172792), the Outstanding Scientific Research Manpower Fund of Fujian Agriculture and Forestry University (xjq201814), the Earmarked Fund for Modern Agro-industry Technology Research System (CARS44-KXJ7), and the Master Supervisor Team Fund of Fujian Agriculture and Forestry University (Rui Guo).

Acknowledgments: We thank all editors and reviewers for their helpful and constructive comments.

Conflicts of Interest: The authors declare that they have no conflict of interest.

\section{References}

1. Fries, I.; Feng, F.; Silva, A.D.; Slemenda, S.B.; Pieniazek, N.J. Nosema ceranae, n. sp. (Microspora, Nosematidae), morphological and molecular characterization of a microsporidian parasite of the Asian honey bee Apis cerana, (Hymenoptera, Apidae). Eur J Protistol. 1996, 32, 356-365, doi: 10.1016/j.jip.2009.05.012.

2. Martín-Hernández, R.; Meana, A.; Prieto, L.; Salvador, A.M.; Garrido-Bailón, E.; Garrido-Bailón, E.; Higes, M. Outcome of colonization of Apis mellifera by Nosema ceranae. Appl Environ Microbiol. 2007, 73, 6331-6338, doi: 10.1128/AEM.00270-07.

3. Mayack, C.; Naug, D. Energetic stress in the honeybee Apis mellifera from Nosema ceranae infection. J Invertebr Pathol. 2009, 100, 185-188, doi: 10.1016/j.jip.2008.12.001.

4. Zheng, S.B.; He, J.F.; Li, Z.G.; Gao, Z.S.; Yu, T.T.; Xu, W.J.; Su, S.K. Effects of Nosema ceranae on the expression of immune gene and hemolymph sugar levels of Apis cerana bees. Entomological Knowledge 2017, 54, 392-399, doi: 10.7679/j.issn. 2095-1353.2017.047. 
5. Senderskiy, I.V.; Timofeev, S.A.; Seliverstova, E.V.; Pavlova, O.A.; Dolgikh, V.V. Secretion of Antonospora (Paranosema) locustae proteins into infected cells suggests an active role of microsporidia in the control of host programs and metabolic processes. PLoS One 2014, 9, 19326203, doi: 10.1371/journal.pone.0093585

6. Schottelius, J.; Schmetz, C.N.; Kock, N.P.; Schüler T.; Sobottka, I., Fleischer, B. Presentation by scanning electron microscopy of the life cycle of microsporidia of the genus Encephalitozoon. Microbes Infect. 2000, 2, 1401-1406, doi: 0.1016/s1286-4579(00)01293-4.

7. Lach, L.; Kratz, M.; Boris, B. Parasitized honey bees are less likely to forage and carry less pollen. J Invertebr Pathol. 2015, 130, 64-71, doi: 10.1016/j.jip.2015.06.003.

8. Dussaubat, C.; Maisonnasse, A.; Crauser, D.; Beslay, D.; Costagliola, G.; Soubeyrand, S.; Kretzchmar, A.; Conte, Y.L. Flight behavior and pheromone changes associated to Nosema ceranae infection of honey bee workers (Apis mellifera) in field conditions. J Invertebr Pathol. 2013, 113, 42-51, doi: 0.1016/j.jip.2013.01.002.

9. Higes, M.; Martín-Hernández, R.; Garrido-Bailón, E.; González-Porto, A.V.; García-Palencia, P.; Meana, A.; Nozal, M.J.D.; Mayo, R.; Bernal, J.L. Honeybee colony collapse due to Nosema ceranae in professional apiaries. Environ Microbiol Rep. 2009, 1, 110-113, doi: 10.1111/j.17582229.2009.00014.x.

10. Bromenshenk, J.J.; Henderson, C.B.; Wick, C.H.; Stanford, M.F.; Zulich, A.W.; Jabbour, R.E.; Deshpande, S.V.; McCubbin, P.E.; Seccomb, R.A.; Welch, P.M.; Williams, T.; Firth, D.R.; Skowronski, E.; Lehmann, M.M.; Bilimoria, S.L.; Gress, J.; Wanner, K.W.; Cramer, R.A.J. Iridovirus and microsporidian linked to honey bee colony decline. PLoS One 2010, 5, e13181, doi: 10.1371/journal.pone.0013181.

11. Chen, Y.P.; Evans, J.D.; Charles, M.; Robin, G.; Michael, Z.; Gundensen-Rindal, D.; Pettis, J.S. Morphological, molecular, and phylogenetic characterization of Nosema ceranae, a microsporidian 
parasite isolated from the European honey bee, Apis mellifera. J Eukaryot Microbiol. 2009, 56, 142147, doi: 10.1111/j.1550-7408.2008.00374.x.

12. Huang, W.F.; Solter, L.F. Comparative development and tissue tropism of Nosema apis and Nosema ceranae. J Invertebr Pathol. 2013, 113, 35-41, doi: 10.1016/j.jip.2013.01.001.

13. Badaoui, B.; Fougeroux, A.; Petit, F.; Anselom, A.; Gorni, C.; Cucurachi, M.; Cersini, A.; Granato, A.; Cardeti, G.; Formato, G.; Mutinelli, F.; Giuffra, E.; Williams, J.L.; Botti, S. RNA-sequence analysis of gene expression from honeybees (Apis mellifera) infected with Nosema ceranae. PLoS One 2017, 12, e0173438, doi: 10.1371/journal.pone.0173438.

14. Dussaubat, C.; Brunet, J.; Higes, M.; Colbourne, J.K.; Lopez J.; Choi, J.H.; Martín-Hernández, R.; Botías, C.; Cousin, M.; McDonnell, C.; Bonnet, M.; Belzunces, L.P.; Moritz, R.F.A.; Conte, Y.L.; Alaux, C. Gut pathology and responses to the microsporidium Nosema ceranae in the honey bee Apis mellifera. PLoS One 2012, 7, e37017, doi: 10.1371/journal.pone.0037017.

15. Panek, J.; Paris, L.; Roriz, D.; Mone, A.; Dubuffet, A.; Delbac, F.; Diogon, M.; Alaoui, H. Impact of the microsporidian Nosema ceranae on the gut epithelium renewal of the honeybee, Apis mellifera. J Invertebr Pathol. 2018, 159, 121-128, doi: 10.1016/j.jip.2018.09.007.

16. Du, Y.; Zhou, D.; Chen, H.; Xiong, C.; Zheng, Y.; Chen, D.; Guo, R. MicroRNA dataset of normal and Nosema ceranae-infected midguts of Apis cerana cerana workers. Data Brief. 2019, 26, 104518, doi: 10.1016/j.dib.2019.104518.

17. Chen, D.; Chen, H.; Du, Y.; Zhou, D.; Geng, S.; Wang, H.; Wan, J.; Xiong, C.; Zheng, Y.; Guo, R. Genome-wide identification of long non-coding RNAs and their regulatory networks involved in Apis mellifera ligustica response to Nosema ceranae infection. Insects 2019, 10, 245, doi: 10.3390/insects 10080245

18. Guo, R.; Chen, D.; Xiong, C.; Zheng, Y.; Hou, C.; Du, Y.; Geng, S.; Wang, H.; Zhou, D.; Guo, Y. Genome-wide identification of circular RNAs in fungal parasite Nosema ceranae. Curr Microbiol. 2018, 29, 1655-1660, doi: 10.1007/s00284-018-1576-Z. 
19. Fu, Z.; Zhou, D.; Chen, H.; Geng, S.; Chen, D.; Zhen, Y.; Xiong, C.; Xu, G.; Zhang, X.; Guo, R. Analysis of highly expressed genes in Apis cerana cerana workers midguts responding to Nosema ceranae stress. Journal of Sichuan University (Natural Science Edition) 2020, 57, 191-198, doi: 10.3969/j.issn.0490-6756.2020.01.029.

20. Xiong, C.; Chen, H.; Geng, S.; Zhou, N.; Zhou, D.; Zhu, Z.; Chen, D.; Zheng, Y.; Xu, G.; Zhang, X.; Guo, R. Expression profile of high-expressing genes and its potential role during Apis cerana cerana infected by Nosema ceranae. Journal of Sichuan University (Natural Science Edition) 2020, 57, 596-604, doi: 10.3969/j.issn.0490-6756.2020.03.030.

21. Xing, W.; Zhou, D.; Long, Q.; Sun M.; Guo, R.; Wang, L. Immune Response of Eastern Honeybee Worker to Nosema ceranae Infection Revealed by Transcriptomic Investigation. Insects 2021, 12, 728, doi: 10.3390/insects12080728.

22. Chen, D, Du, Y, Chen, H, Fan, Y.; Fan, X.; Zhu, Z.; Wang, J.; Xiong, C.; Zheng, Y.; Hou, C.; Diao, Q.; Guo, R. Comparative identification of microRNAs in Apis cerana cerana workers' midguts in response to Nosema ceranae invasion. Insects 2019, 10, 258-280, doi: 10.3390/insects10090258.

23. Chen, Y.; Evans, J.D.; Smith, I.B.; Pettis, J.S. Nosema ceranae is a long-present and wide-spread microsporidian infection of the European honey bee (Apis mellifera) in the United States. J Invertebr Pathol. 2008, 97,186-188, doi: 10.1016/j.jip.2007.07.010.

24. Langmead, B.; Salzberg, S.L. Fast gapped-read alignment with Bowtie 2. Nat. Methods 2012, 9, 357-359, doi: 10.1038/nmeth.1923.

25. Fu, Z.M.; Zhou, D.D.; Chen, H.Z.; Geng, S.H.; Chen, D.F.; Zheng, Y.Z.; Xiong, C.L.; Xu, G.J.; Zhang, X.; Guo, R. Analysis of highly expressed genes in Apis cerana cerana workers' midguts responding to Nosema ceranae stress. Sichuan Univ 2020, 57, 191-198, doi: 10.3969/j.issn.04906756.2020.01.02. 
26. Chen, Y.; Carthy, D.M.; Robinson, M.; Robinson, M.; Smyth, G. edgeR: a Bioconductor package for differential expression analysis of digital gene expression data. Bioinformatics. 2010, 26, 139140, doi: 10.1093/bioinformatics/btp616.

27. Jia, Y.; Lin, F.; Hongkun, Z.; Zhang, Y.; Chen, J.; Zhang, Z.; Wang, J.; Li, S.; Li, R.; Bolund L.; Wang L. WEGO: a web tool for plotting GO annotations. Nucleic Acids Res. 2005, 2, 293-297, doi: 10.1093/nar/gk1031.

28. Kanehisa, M.; Araki, M.; Goto, S.; Hattori, M.; Hirakawa, M.; Itoh, M.; Katayama, T.; Kawashima, S.; Okuda, S.; Tokimatsu, T.; Yamanishi, Y. KEGG for linking genomes to life and the environment. Nucleic Acids Res. 2008, 36, D480-484, doi: 10.1093/nar/gkm882.

29. Du, Y.; Fan, X.; Jiang, H.; Wang, J.; Feng, R.; Zhang, W.; Yu, K.; Long, Q.; Cai, Z.; Xiong, C.; Zheng, Y.; Chen, D.; Fu, Z.; Xu, G; Guo, R. MicroRNA-mediated cross-kingdom regulation of Apis mellifera ligustica worker to Nosema ceranae. Scientia Agricultura Sinica 2021, 54, 1805-1820, doi: 10.3864/j.issn.0578-1752.

30. Fan, X.; Du, Y.; Zhang, W.; Wang, J.; Jiang, H.; Fan, Y.; Feng, R.; Wang, J.; Zhou, Z.; Xiong, C.; Zheng, Y.; Chen, D.; Guo, R. Omics analysis of Nosema ceranae miRNAs involved in gene expression regulation in the midgut of Apis mellifera ligustica workers and their regulatory networks. Acta entomologica sinica 2021, 64, 187-204, doi: 10.16380/j.kcxb.2021.02.006.

31. Geng, S.; Zhou, D.; Fan, X.; Jiang, H.; Zhu, Z.; Wang, J.; Fan, Y.; Wang, X.; Xiong, C.; Zheng, Y.; Fu, Z.; Chen, D.; Guo, R. Transcriptomic analysis reveals the molecular mechanism underlying Nosema ceranae infection of Apis mellifera ligustica. Acta entomologica sinica 2020, 63, 294-308, doi: 10.16380/j.kcxb.2020.03.006.

32. Livak, K.J.; Schmittgen, T.D. Analysis of relative gene expression data using real-time quantitative PCR and the 2(-Delta Delta C(T)) Method. Methods 2001, 25, 402-408, doi: 10.1006/meth.2001.1262. 
33. Guo, R.; Chen, D.; Huang, Z.; Liang, Q.; Xiong, C.; Xu, X.; Zheng, Y.; Zhang, Z.; Xie, Y.; Tong,

X.; Hou, Z.; Jiang, L.; Dao, C. Transcriptome analysis of Ascosphaera apis stressing larval gut of Apis cerana cerana. Acta microbiologica sinica 2017, 57, 1865-1878, doi: 10.13343/j.cnki.wsxb.20160551.

34. Chen, D.; Guo, R.; Xiong C.; Liang, Q.; Zheng, Y.; Xu, X.; Huang, Z.; Zhang, Z.; Zhang, L.; LI, W.; Tong, X.; Xi, W. Transcriptomic analysis of Ascosphaera apis stressing larval gut of Apis mellifera ligustica (Hyemenoptera: Apidae). Acta entomologica sinica 2017, 60, 401-411, doi: 10.16380/j.kcxb.2017.04.005.

35. Xiong, C.; Du, Y.; Feng, R.; Jiang, J.; Shi, X.; Wang, H.; Fan, X.; Wang, J.; Zhu, Z.; Fan, Y.; Chen, H.; Zhou, D.; Zheng, Y.; Chen, D.; Guo, R. Differential expression pattern and regulation network of microRNAs in Ascosphaera apis invading Apis cerana cerana 6-day-old larvae. Acta microbiologica sinica 2020, 60, 992-1009, doi: 10.13343/j.cnki.wsxb.20190421.

36. Pérez, P.; Cansado, J. Cell integrity signaling and response to stress in fission yeast. Curr Protein Pept Sci. 2010, 11, 680-692, doi: 10.2174/138920310794557718.

37. Liu, H.; Li, M.; Cai, S.; He, X.; Shao, Y.; Lu, X. Ricin-B-lectin enhances microsporidia Nosema bombycis infection in BmN cells from silkworm Bombyx mori. Acta Biochim Biophys Sin (Shanghai). 2016, 48, 1020-1057, doi: 10.1093/abbs/gmw093.

38. Qi. Studies on the functional genome of Nosema bombycis-localization and functional analysis on Ricin B-lectin NbRBL3 of Nosema bombycis. Chongqing: Southwest University 2014, 1-88.

39. Long, M.; Tan, Y.; Yu, B.; Pan, G.; Zhou, Z. Expression of Nosema bombycis polar tube protein 1 in lepidopteran sf9 cells and its effect on microsporidian proliferation. J Invertebr Pathol. 2011, 30056-2, doi: 10.1016/j.jip.2020.107350.

40. Wu, Z.; Li, Y.; Pan, J.; Zhou, Z.; Xiang, Z. SWP25, a novel protein associated with the Nosema bombycis endospore. $J$ Eukaryot Microbiol. 2009, 56, 113-118, doi: 10.1111/j.15507408.2008.00375.x. 
41. Yang, D.; Pan, L.; Peng, P.; Dang, X.; Li, C.; Li, T.; Long, M.; Chen, J.; Wu, Y.; Du, H.; Luo, B.; Song, Y.; Tian, R.; Luo, J.; Zhou, Z.; Pan, G. Interaction between SWP9 and polar tube proteins of the microsporidian Nosema bombycis and function of SWP9 as a scaffolding protein contribute to polar tube tethering to the spore wall. Infect Immun. 2017, 85, e00872-16. doi: 10.1128/IAI.0087216.

42. Yang, D.; Pan, L.; Chen, Z.; Du, H.; Luo, B.; Luo, J.; Pan, G. The roles of microsporidia spore wall proteins in the spore wall formation and polar tube anchorage to spore wall during development and infection processes. Exp Parasitol. 2018, 187, 93-100. doi: 10.1016/j.exppara.2018.03.007.

43. Long, M.; Tan, Y.; Liu, K.; Wu, Y.; Lü, Q.; Pan, G.; Zhou, Z. Expression of Polar Tube Protein 1 (NbPTP1) from Nosema bombycis in Drosophila S2 cell lines and its glycosylation. Chinese Journal of Biotechnology 2018, 34,1460-1468, doi: 10.13345/j.cjb.170478.

44. Cabib, E.; Sburlati, A.; Bowers, B.; Silverman, S.J. Chitin synthase 1, an auxiliary enzyme for chitin synthesis in Saccharomyces cerevisiae. J Cell Biol. 1989, 108, 1665-1672, doi: 10.1083/jcb.108.5.1665.

45. Huang, Q.; Chen, Y.; Wang, R.; Cheng, S.; Evans, J. Host-parasite interactions and purifying selection in a microsporidian parasite of honey bees. PloS One 2016, 11, e0147549, doi: 10.1371/journal.pone.0147549.

46. Chen, X.; Peng, X.; Li X.; Kye,i B.; Shang, R.; Qi, J.; Zhang, Y.; Xu, L.; Sheng, Z. Cloning, sequence structure analysis and prokaryotic expression of Nosema bombycis hexokinase gene. Acta Sericologica Sinica 2018, 044, 70-76, doi: 10.13441/j.cnki.cykx.2018.01.009.

47. Zhang, Z.; Qi, J.; Shang, R.; Chen, H.; Zhang, Y.; Sheng, Z. Cloning and expression characteristics of pyruvate kinase gene of Nosema bombycis. Acta Sericologica Sinica 2019, 045, 212-217, doi: 10.13441/j.cnki.cykx.2019.02.008. 
48. Yao, Q.; Yang, Y.; Cui, L.; Su, H.; Li, R.; Lu, G. Elementary analysis of 6-phosphofructokinase gene of Xanthomonas campestris. Genomics and Applied Biology 2010, 29, 1047-1054, doi: 10.3969/gab.029.001047.

49. Dermauw, W.; Leeuwen, T.V. The ABC gene family in arthropods comparative genomics and role in insecticide transport and resistance. Insect Biochem Mol Biol. 2014, 45, 89-110, doi: 10.1016/j.ibmb.2013.11.001.

50. He, Q.; Vossbrinck, C.R.; Yang, Q.; Meng, X.Z.; Luo, J.; Pan, G.Q.; Zhou, Z.Y.; Li, T. Evolutionary and functional studies on microsporidian ATP-binding cassettes: Insights into the adaptation of microsporidia to obligated intracellular parasitism. Infect Genet Evol. 2019, 68, 136144, doi: 10.1016/j.meegid.2018.12.022.

51. Paldi, N.; Glick, E.; Oliva, M.; Zilberberg, Y.; Aubin, L.; Pettis, J.; Chen, Y.; Evans, JD. Effective gene silencing in a microsporidian parasite associated with honeybee (Apis mellifera) colony declines. Appl Environ Microbiol. 2010, 76, 5960-5964, doi: 10.1128/AEM.01067-10. 